\title{
FEATURES OF THE NIH ATLAS SMALL ANIMAL PET SCANNER AND ITS USE WITH A COAXIAL SMALL ANIMAL VOLUME CT SCANNER
}

\author{
Jürgen Seidel ${ }^{+}$, Juan J. Vaquero*, Javier Pascau", Manuel Desco*, \\ Calvin A. Johnson ${ }^{+}$and Michael V. Green ${ }^{+}$ \\ ${ }^{+}$National Institutes of Health, Bethesda, MD, U.S.A. \\ *Hospital Universitario Gregorio Marañón, Madrid, Spain
}

\begin{abstract}
ATLAS (Advanced Technology Laboratory Animal Scanner), a small animal PET scanner designed to image animals the size of rats and mice, is about to enter service on the NIH campus in Bethesda, Maryland. This system is the first small animal PET scanner with a depth-ofinteraction capability and the first to use iterative resolution recovery algorithms, rather than conventional filtered back projection, for "production" image reconstruction.

ATLAS is also proximate to, and co-axial with, a high resolution small animal CT scanner. When fully integrated, spatially registered PET and CT images of each animal will be used to correct the emission data for radiation attenuation and to aid in target identification. In this report we describe some of the technical and finctional features of this system and illustrate how these features are used in an actual small animal imaging study.
\end{abstract}

\section{INTRODUCTION}

A small animal PET scanner should have high sensitivity and high and uniform spatial resolution across a field-ofview larger than the largest object to be imaged. Moreover, if such a device is to yield quantitatively accurate estimates of regional organ radioactivity, it must provide the ability to correct the emission data for attenuation and other perturbing effects. Finally, if the system is to be used routinely by scientists and technologists without a physics or engineering background, the user interface and the capabilities provided through the interface must be transparent to the user, i.e. "user-friendly". We have sought to meet these requirements (1) by designing a small ring diameter, high sensitivity, high resolution, depth-of-interaction (DOI) PET scanner for imaging animals up to $6 \mathrm{~cm}$ in diameter; (2) by providing this system with a user interface that allows data to be acquired, reconstructed and viewed with only a few mouse clicks and parameter entries and (3) by eventually performing attenuation correction of the emission data using CT images obtained with a recently installed high resolution, small animal, volume imaging CT scanner (Im Tek, Inc., microCAT II, Knoxville, Tenn.) placed immediately behind and co-axial with ATLAS. We describe in more detail below some of these technical and user-oriented features.

\section{METHODS AND MATERIALS}

\subsection{Design Features}

ATLAS consists of 18 LGSO/GSO (Hitachi) phoswich detector modules arranged around a ring $11.8 \mathrm{~cm}$ in diameter. Each module is comprised of a $9 \times 9$ array of 2 $\mathrm{mm}$ square $\times 15 \mathrm{~mm}$ deep phoswich elements $(2.25 \mathrm{~mm}$ pitch), each of which is composed of a $7 \mathrm{~mm}$ long LGSO crystal optically glued end-on to an $8 \mathrm{~mm}$ long GSO crystal. The GSO end of the crystal bundle is optically glued to a miniature PSPMT (Hamamatsu R7600-C8). The scintillator (or depth)-of-interaction is identified by measuring the decay time (LGSO: $40 \mathrm{~ns}$, GSO: $60 \mathrm{~ns}$ ) of the light pulse of each event [1]. With this technique it is possible to have a substantial total crystal depth (15 mm) while at the same time having apparently short crystals (7 $\mathrm{mm}$ of LGSO and $8 \mathrm{~mm}$ of GSO) at small ring diameter. The small ring diameter, in tum, further improves sensitivity by increasing the system solid angle.

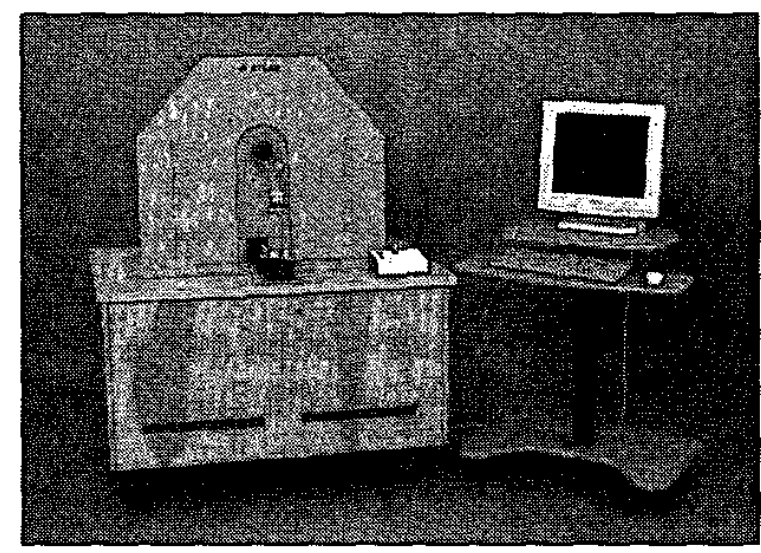

Figure 1. The NIH ATLAS small animal PET seanner.

The aperture of the ATLAS scanner shown in Figure 1 is $8 \mathrm{~cm}$ in diameter with a useful transverse field-ofview of $6 \mathrm{~cm}$ and an axial field-of-view of $2 \mathrm{~cm}$. The 
ATLAS detector arcay inside the gantry cover can be "wobbled" at rates up to eight revolutions/sec during data acquisition to improve spatial sampling.

The animal bed shown in Figure 1 can be moved manually into and out of the aperture by joystick (mounted on the white box on corner of gantry enclosure) or under computer control. Typically, the animal and bed are advanced manually until the desired imaging region is under a laser positioning system (not shown) just outside the scanning aperture. The computer then automatically advances the bed the remaining distance to place the target region exactly at the center of the axial field-of-view.

With the animal in place, data acquisition can begin The eighteen ATLAS detector modules are combined mechanically and electrically into groups of three to form a total of six sectors. The signals originating from the ATLAS sectors are fed to a data acquisition system [2] supplied by A \& D Precision Co. (Newton, MA 02460, USA). The system consists of six charge integrating ADC modules with custom modifications to facilitate identification of scintillation decay times, one scaler module, a custom coincidence logic controller, and a high-speed PCI- bus interface card. The controller detects coincidences between sectors and initiates signal integration and ADC read out. The data are collected by two alternating $128 \mathrm{kB}$ memory buffers on the PCI-bus card in a dual-processor PC operating under the Linux OS. The data acquisition system, power supplies, motor controllers and drivers, and computer are all housed in the wood-paneled enclosure shown in Figure 1.

\subsection{Functional Features}

ATLAS is intended for use by scientists and technologists without extensive imaging experience. Accordingly, the ATLAS user interface is designed to allow users with minimal training to perform all of the functions necessary to carry out a small animal PET imaging study, data acquisition, image reconstruction and image analysis and display, with only a few mouse clicks and parameter entries.

The ATLAS user interface supports static, dynamic, whole-body data acquisitions and, if necessary, transmission scans using a local Ge-68 ring. During setup, the user can view a real-time axial projection image of the field-of-view so that animals already labeled with tracer can be positioned in the field-of-view based on the apparent activity distribution. When data acquisition is finally started, this persistence image becomes a cumulating projection image so that the user can be continuously apprised of apparent image quality, animal movement, etc. during data acquisition.

When data acquisition is over, the user can initiate two kinds of image reconstructions: filtered back projection (FBP) on the local ATLAS computer or iterative, resolution recovery $3 \mathrm{D}$ OSEM reconstruction on a remote, very large Beowulf-type computer cluster [3]. Either of these two choices are selected from short menus where advantage is taken of pre-selected default values for filter types and degree of smoothing (FBP) and iteration number and other parameters required for 3D OSEM reconstruction. If remote reconstruction is selected, ATLAS can again be used for data collection once the acquired data set has been sent by network to the computer cluster. During off-site reconstruction, the status of the reconstruction is periodically updated and displayed to the user by the ATLAS user interface. When completed, the reconstructed images are automatically returned to the ATLAS computer over the network for display and analysis.

ATLAS currently supports a variety of visualization and analysis options that include display of static and dynamic images in stack or montage modes, simultaneous orthogonal viewing of 3D image stacks ("orthoviewing"), ROI definition, time-activity curve generation, volume rendering, movie display and simultaneous viewing of spatially registered dual-modality imaging studies. Software has been developed [4], but not yet installed, that will allow multi-modality software image registration. These capabilities will be fully exploited when ATLAS and the coaxial small animal CT scanner shown in Figure 2 are fully integrated. Animals will eventually be able to be moved mechanically from one machine to the other in spatial registration without manual intervention (as is now the case) although software registration of these data, and of other study types, e.g. MRI, may be equally effective [4].

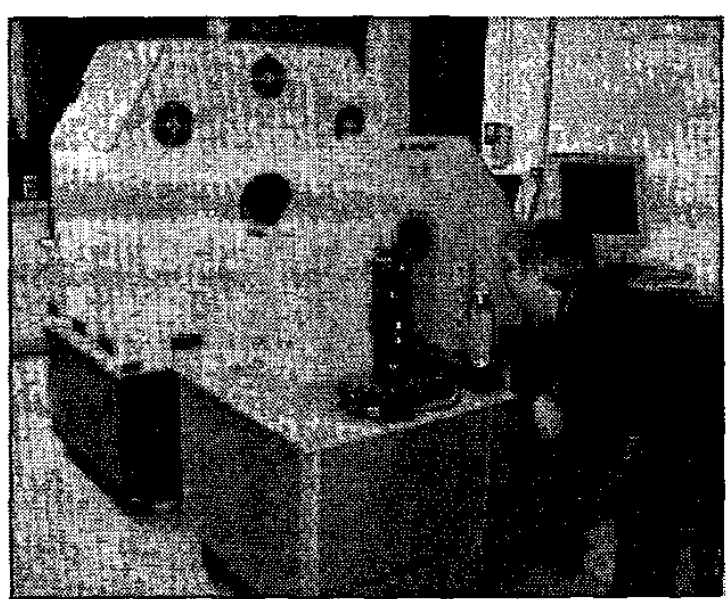

Figure 2. Small animal CT scanner located behind and coaxial with the ATLAS scanner.

\section{RESULTS}

\subsection{Imaging Performance}

The three major technical design goals of the ATLAS system were verified by measurements made on the completed system. First, the system was designed to exhibit a high central point source sensitivity (CPSS) 
through use of relatively long phoswich crystal elements and a small ring diameter. CPSS was measured to be $1.8 \%$ for an energy window of $250-650 \mathrm{keV}$.

Second, ATLAS was intended to exhibit a spatial resolution compatible with imaging animals the size of rats and mice $(<2 \mathrm{~mm})$. The apparent FWHM width of a $0.5 \mathrm{~mm} \mathrm{Na}-22$ source located at the geometric center of the field-of-view was $1.8 \mathrm{~mm}$.

Third, radial resolution degraded by $25 \%$ across the central $3 \mathrm{~cm}$ of the effective field-of-view and by $39 \%$ across the full $6 \mathrm{~cm}$ field-of-view, the limit of the scanning field.

\subsection{Functional Performance}

In order to illustrate the functional capabilities of ATLAS (and the CT scanner), a rat imaging study was carried out using several of the ATLAS user interface functions described in this report. In particular, a $325 \mathrm{gm}$ rat was injected with $4.5 \mathrm{mCi}$ of F-18 labeled FDG and 30 minutes allowed for uptake. At 30 minutes the animal was sacrificed to eliminate animal movement and the need for extensive life support during imaging.

The animal was placed on the ATLAS imaging bed and "whole-body" imaging selected through the user interface. Once acquisition was initiated, the animal was automatically advanced in ten steps through the scanning aperture to acquire overlapping data sets that spanned about $50 \%$ of the whole body. These data sets were corrected for decay but not for attenuation or other effects. The data sets were then transferred to the computer cluster through the user interface and reconstruction was performed off site using the OSEM algorithm with resolution recovery. The reconstructed images were then returned to ATLAS over the network.

After the FDG scan was completed, the animal bed with animal attached was removed from ATLAS and manually transferred to the CT scanner. A 200 micronresolution volumetric $\mathrm{CT}$ scan was then acquired covering approximately the same fraction of the body as the ATLAS scan. These CT data were reconstructed locally using a hardware Feldkamp reconstructor provided with the scanner.

Since direct mechanical registration of ATLAS and CT image data was not (yet) possible, the two data sets were spatially registered off-line and superimposed with previously validated software [4]. Spatially registered and overlaid sagittal and coronal section PET and CT images covering about half the body length are shown in Figures 3 and 4, respectively. The principal features visible in these Figures are the skull and spine (by CT) and the heart and brain (by FDG PET). The rendering technique (brightness/hue), adjusted to accentuate registration accuracy, saturates color and suppresses spatial detail within FDG-intense regions, e.g. heart and brain.

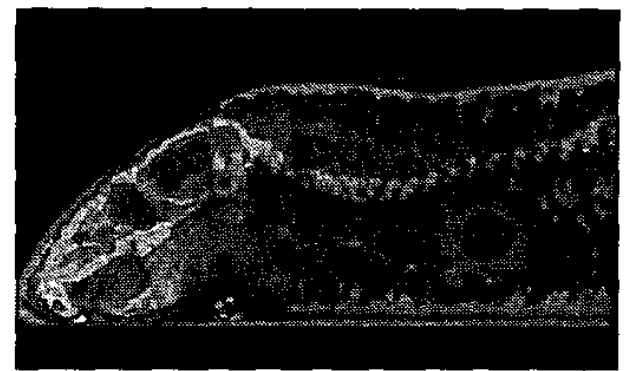

Figure 3. Spatially registered and superimposed near midline sagittal CT and ATLAS FDG PET images of the rat. Note the (FDG) brain fit within the bony (CT) outline of the brain case and the heart resting against the ribs.

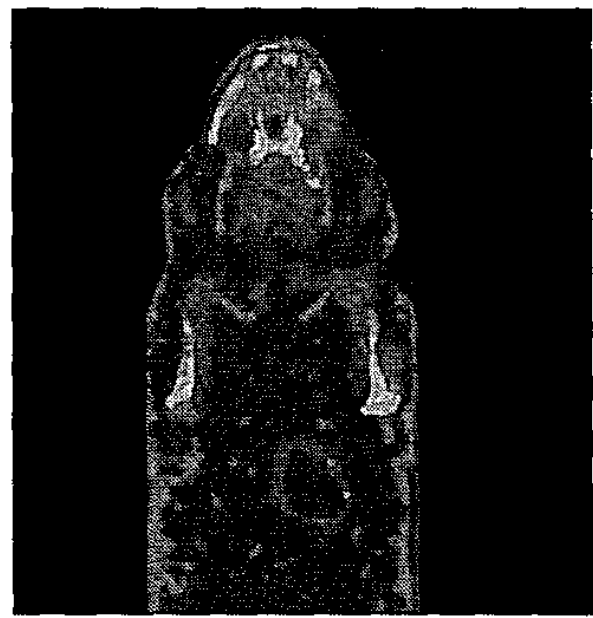

Figure 4. Spatially registered and superimposed anterior coronal section CT and ATLAS FDG PET images of the rat. Note the heart and ribs in the chest and the faint outline of muscle adjacent to the leg bones.

\section{DISCUSSION AND CONCLUSIONS}

The sensitivity of the ATLAS small animal PET scanner is higher, by design, than several other small animal PET scanners. Although ATLAS has only a $2 \mathrm{~cm}$ axial fieldof-view, its sensitivity of $2.7 \%$ (low energy threshold) is higher than the $28 \mathrm{~cm}$ axial field-of-view QUAD-HIDAC [5] $(1.5 \%)$ when no correction is made for scattered radiation, three times higher than the UCLA microPET [6] (1.8\% ATLAS vs. $0.56 \%$ microPET, $250-650 \mathrm{keV}$ window) and almost as sensitive as the commercial microPET P4 [7] (1.8\% ATLAS vs. $2.25 \%$ P4, 250-650 $\mathrm{keV}$ window) despite the greater $(7.8 \mathrm{~cm})$ axial extent of the P4. At the same time, ATLAS exhibits a (central) spatial resolution of $1.8 \mathrm{~mm}$, a value comparable to the UCLA microPET $(1.8 \mathrm{~mm})$, better than the commercial microPET P4 $(2.3 \mathrm{~mm})$ but somewhat poorer than the stated operational resolution of the QUAD-HIDAC (1.5 $\mathrm{mm}$ ). At the same time, the degradation in radial 
resolution with increasing radial position exhibited by ATLAS is no worse than scintillator-based machines having shorter crystals, larger ring diameters, or both $[6,7]$.

The functional features of ATLAS include simple menus for initiating all of the common data acquisition types and image visualization and analysis routines needed by a typical user to acquire and view a small animal PET imaging study. In addition, the ATLAS user interface supports both local FBP reconstruction and 3D OSEM resolution recovery image reconstruction on a very large-scale (Beowulf-type) computer cluster at a site remote from the imaging laboratory. Together, these features are expected to provide the user with a substantial imaging resource for performing a wide variety of experimental PET imaging protocols.

The use of ATLAS in conjunction with a separate small animal CT scanner offers a number of additional experimental and research opportunities. Energy-scaled CT images are now already in use to correct PET emission data for radiation attenuation in human studies [8] and should be equally effective in producing nearly noise-free attenuation corrections for small animal PET images. Similarly, it has been demonstrated in human studies [9] that PET/CT image overlays can materially aid the observer in identifying and characterizing the site of radiophannaceutical accumulation, a feature that may be of particular value when trying to assess the biodistribution of new radiopharmaceuticals in small animals. In each of these cases, a CT scanner separate from the ATLAS gantry is advantageous since new emission data can be acquired while another animal is undergoing CT imaging.

Several interesting research questions can also be addressed if spatially registered CT and PET image data are available. For example, spatially registered $\mathrm{CT}$ images might be used to spatially modulate the positron range point spread function as tissue density varies from point to point throughout the body. This spatially deformable range function could, in turn, be incorporated into the model supplied to the iterative reconstruction code to remove not just the "blur" due to positron range but also the residual blur due to positron range variations.

The technical and functional features designed into the ATLAS small animal PET scanner were chosen to give the user an easy-to-operate instrument with substantial imaging performance while providing research capabilities useful to the imaging scientist. It is anticipated that the ATLAS machine will continue to evolve as user experience accumulates and new technical and functional capabilities are added.

\section{ACKNOWLEDGEMENTS}

This work utilized the high-performance computational capabilities of the Biowulf/LoBoS3 cluster at the National Institutes of Health, Bethesda, MD.
The authors are deeply indebted to J. Sullivan, P. Fitze, J. Powell, F. Sharpnack II, and C. Toms of the Mechanical Instrument Design \& Fabrication Section and to B. Chidakel, Computer \& Electronics Section, Office of Research Services, NIH for their help with the ATLAS project. The authors also thank $R$. Ford, Division of Engineering Services, NIH, and F. J. Barbosa and W. Gunning, Thomas Jefferson National Accelerator Facility. We also gratefully acknowledge the assistance of $\mathbf{V}$. Zavarzin, A \& D Precision Co., for customizing the data acquisition system to our specifications.

This work was supported in part by projects FIS 00/0036 and III PRICIT Comunidad de Madrid, Spain.

\section{References}

[1] J. Seidel, J. J. Vaquero, S. Siegel, W. R. Gandler, and M. V. Green, "Depth Identification Accuracy of a Three-Layer Phoswich PET Detector Module", IEEE Trans Nucl Sci, vol. 46, no. 3, pp. 485-490, 1999.

[2] V. G. Zavarzin and W. A. Earle, "A 500k Event/s 12-Bit ADC System with high-speed buffered PCI Interface," in Conference Record of the 1998 IEEE Nuclear Science Symposium, paper N20-25.

[3] C. A. Johnson, J. Seidel, R. E. Carson, W. R. Gandler, A. Sofer, M. V. Green, and M. E. Daube-Witherspoon, "Evaluation of 3D Reconstruction Algorithms for a Small Animal PET Camera", IEEE Trans Nucl Sci, vol. 44, no. 3, pp. 1303-1308, 1997.

[4] J.J. Vaquero, M. Desco, J. Pascau, A. Santos, I. Lee, J. Seidel and M.V. Green, "PET, CT and MR lmage Registration of the Rat Brain and Skull", IEEE Trans Nuc Sci, vol. 48, no.4, pp. 1440-1445, 2001.

[5] J. Missimer, M. Honer, S. Ametamey and P.A. Schubiger, "Perfornance of 16-Module Quad-Hidac PET Camera", HiRes 2001 Proceedings, Rockville, MD, September, 2001. On website: http://www.ornl.gov/HiRes2001/

[6] A. F. Chatziioannou, S. R. Cheny, Y. Shao, R. W. Silverman, K. Meadors, T. H. Farquhar, M. Pedarsani, and M. E. Phelps, "Performance Evaluation of MicroPET: a High-resolution Lutetium Oxyorthosilicate PET Scanner for Animal Imaging", J Nucl Med, vol. 40, no. 7, pp. 1164-1175, 1999.

[7] Y. C. Tai, A. Chatziioannou, S. Siegel, J. Young, D. Newport, R.N. Goble, R. E. Nutt and S.R. Cherry, "Performance Evaluation of the microPET P4: a PET system dedicated to animal imaging", Phys. Med. Biol., vol. 46, pp. 1845-1862, 2001.

[8] R. Bar Shalom, Z. Keidar, A. Engel, M. Mor, D. Gaitini, "A New Combined Dedicated PET/CT System in the Evaluation of Cancer Patients", J Nucl Med, vol. 42, pp. 34P, 2001.

[9] C.C. Meltzer, M.A. Martinelli, T.Beyer, P.E. Kinahan, M. Charron, B. McCook, D.W.Townsend, "Whole body PET Imaging in the Abdomen: Value of Combined PET/CT", J Nucl Med, vol. 43, no. 5, pp.35P, 2001. 